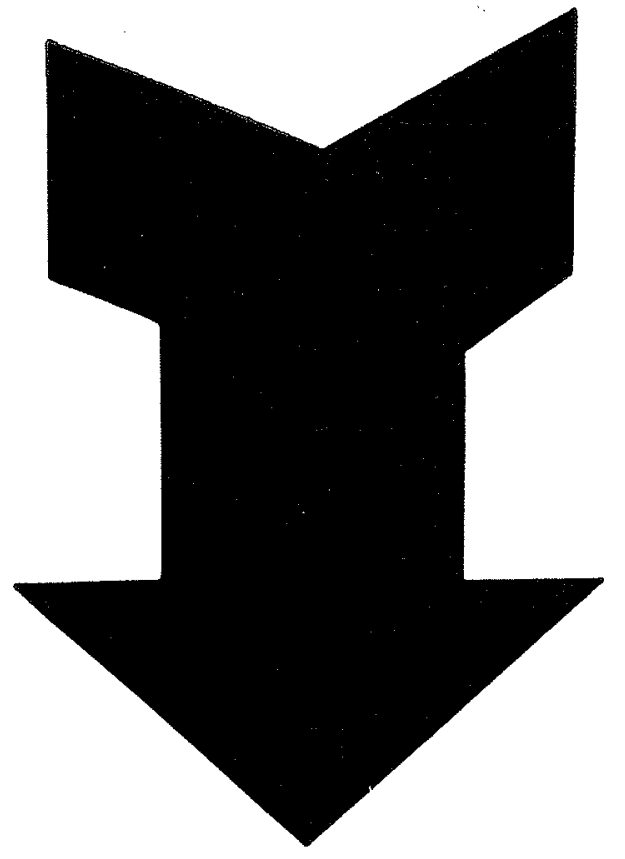

\title{
FONCTIONNEMENT ET ENTRETIEN DES FOSSÉS D'ASSAINISSEMENT DANS LE MARAIS CHARENTAIS
}
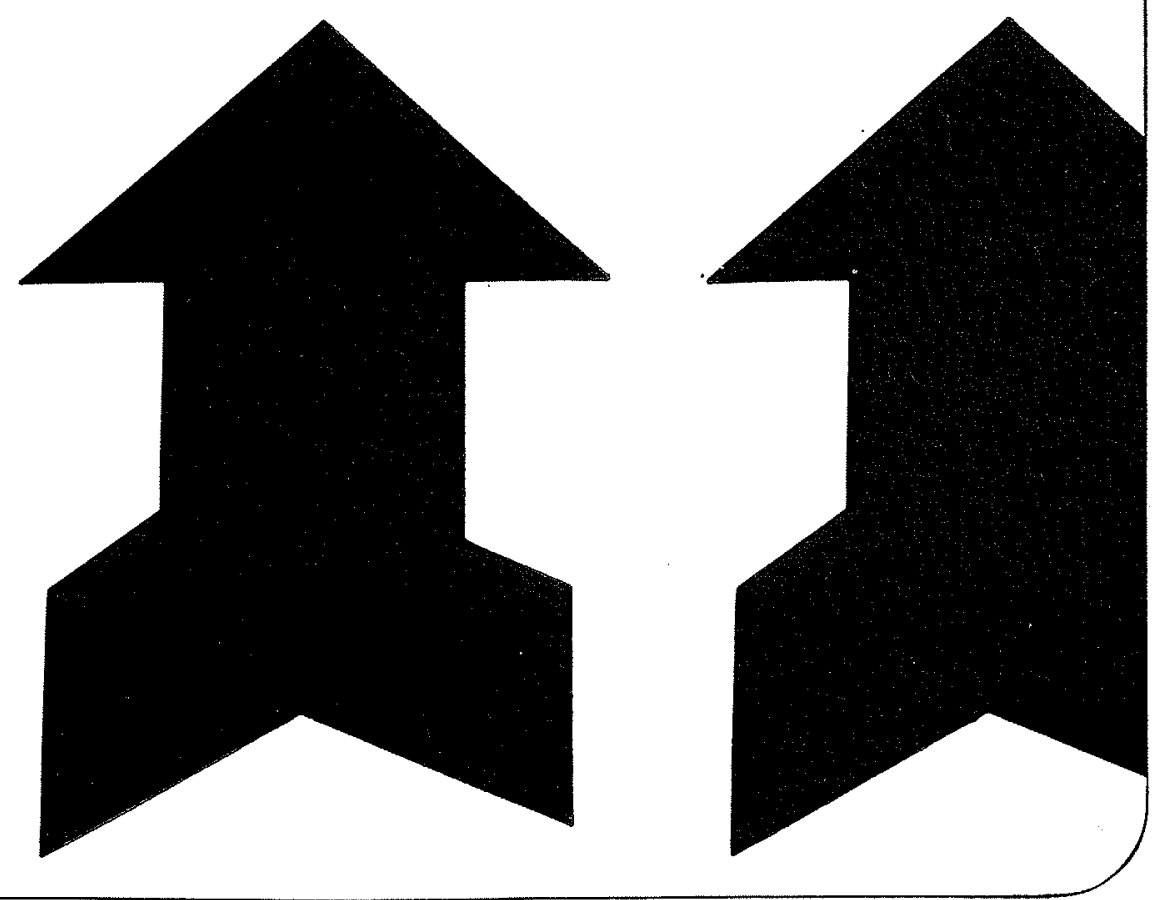

\section{Le cadre géographique et les données hydrauliques}

Le problème de l'entretien des réseaux d'assainissement en Charente-Maritime est particulièrement important, puisque sur les 450000 hectares que comporte le département, on compte 80000 hectares de marais et 8000 kilomètres de fossés el canaux.

Avant d'aborder l'étude de ce problème, il est nécessaire de définir bricvement le cadre géographique dans lequel il se situe, de donner un aperesu sur ce que l'on appelle le marais et de préciser la structure et le fonctionnement hydraulique de son réseau d'assainissement.

\subsection{Le marais.}

Le terme marais désigne des zones qui ont en commun d'être basses et très plates, mais qui peuvent avoir des origines et partant des situations bien distinctes.

\subsubsection{LEs Marais côtrers}

Le littoral atlantique a subi à l'époque pliocène une série de cassures qui ont entrainé la formation de golfes profonds semés d'îles et bordés par

\footnotetext{
* Ingénieur du Génie Rural, des Eatix et des Forêts, à la Direction départementale de l'Agriculture de la CharenteMaritime.
}

endroits de falaises abruples : le golfe du Poitou, a l'embouchure acluelle de la Sèvre Niortaise, le golfe de la région de Rochefort, à l'estuaire de la Charente, et d'autres de moindre imporlance. Les aiternances de transgressions et régressions marines ont ensuite provoqué, depuis l'époque flandrienne et jusqu'à la période historique, une sédimentation intense et le comblement de ces golfes par une argile bleue à Scrobicularia plana appelée bri. A l'heure actuelle, les marais côtiers se présentent comme d'immenses étendues plates et sans arbres vouées à la prairie permanente. Leur cote moyenne $(2,60 \mathrm{~m}$ à $3,00 \mathrm{~m}$ NGF) est inférieure à celle des hautes mers des marées de syzigie $(+3,50 \mathrm{~m} \mathrm{NGF})$; les points les plus hauts sont généralement situés en bordure de mer et la pente du terrain, qui est donc inverse du sens d'éconlement de l'eau, est extrêmement faible (2 à $3 \mathrm{~cm}$ par kilomètre).

Au milieu de ces étendues plates, bordées par les anciennes falaises marines calcaires, subsistent les illes qui parsemaient le golfe comme autant de buttes témoins dont la toponymie rappelle bien l'origine (l'île d'Albe, l'île d'Elle, etc.).

\subsubsection{LES MARAIS DE FOND DE VALLÉE}

Les fleuves côtiers et leurs affluents qui sillonnent le département ont en général des pentes très faibles et leurs vallées, étroites vers l'amont puis s'évasant largement en arrivant à l'aval, ont été colmatées à l'époque quaternaire par d'épaises alluvions tourbo-argileuses d'origine fluviatile. Les marais de vallée se présentent donc comme des bandes étirées le long des cours d'eau qui offrent des 
paysages variés, souvent boisés. La cote des terrains varie entre 20 et $5 \mathrm{~m} \mathrm{NGF}$, ce qui les soustrait à l'action directe de la marée.

La mise en valeur systématique de ces zones marécageuses remonte au $X^{*}$ siècle et se poursuit encore de nos jours.

L'aspect des réseaux d'assainissement est évidemment très différent d'un type de marais à l'autre.

Dans les marais de fond de vallée, qui ne représentent que 15 à $20 \%$ de la superficie totale du marais Charentais, le problème à résoudre est celui de la protection contre les crues. La rivière, qui sert d'émissaire principal, est donc calibrée en conséquence et recoit les collecteurs secondaires drainant les terrains. Parfois, elle a été endiguée de manière à pouvoir transiter les fortes crues sans débordement.

La structure et le fonctionnement de ces réseaux de fond de vallée n'offrent aucune caractéristique spéciale et le seul élément à retenir sous l'angle de leur entretien est que les fossés et canaux sont fréquemment bordés d'arbres.

I.a mise en valeur des marais cotiers qui représcntent plus de $80 \%$ de l'ensemble du marais Charentais est plus complexe.

Les ouvrages réalisés comportent :

- une digue de protection contre la mer qui se referme sur les terres hautes ou sur la digue d'un marais voisin préexistant;

- un réseau d'assainissement comprenant en général un canal principal débouchant à la mer à travers la digue par l'intermédiaire d'un vannage mancuvré manuellement ou automatiquement en fonction du niveau de la marée, des canaux secondaires et un réseau de collecteurs tertiaires dont le type habituel est le fossé dit de 10 pieds, soit $3 \mathrm{~m}$ à $3,50 \mathrm{~m}$ d'ouverture.

C'est l'entretien de ces réseaux que traitera le plus Ionguement cet exposé, ne serait-ce qu'en raison de leur importance relative dans le département : on estime à $7600 \mathrm{~km}$ la longueur de fossés de 10 pieds en service, soit environ $100 \mathrm{~m}$ par hectare de marais.

\subsection{Les réseaux.}

Le tracé et les caractéristiques dimensionmelles des fossés tertiaires et de la plupart des canaux secondaires n'ont sans doute jamais été et ne sont toujours pas dictés par des considérations hydrauliques. Les facteurs traditionnels, dont le poids se fait toujours sentir, sont essentiellement:

- la structure du parcellaire;

- le mode d'exploitation : le fossé tertiaire, étant utilisé comme clôture, doit avoir une largeur minimale (ici $3 \mathrm{~m}$ ), ce qui permet également de constituer de la sorte pour la saison sèche, une réserve d'eau qui est également destinée à l'abreuvement des animaux.

Il est plus difficile de se faire une idée des critères retenus par nos anciens pour déterminer les caractéristiques fondamentales des canaux principaux, à savoir :

- la pente, c'est-à-dire en définitive, le terrain pouvant être considéré comme plat, la cote du radier de l'ouvrage d'évacuation à la mer;
- la section du canal et celle de l'ouvrage de sortie.

Un examen rapide montre d'ailleurs des differences de régime hydraulique considérable d'un réseau à l'autre et accessoirement des insuffisances manifestes de certains d'entre eux. On a donc recherché une méthode permettant de déterminer les caractéristiques à donner aux ouvrages de la manière la plus rigoureuse possible.

\section{a) Collecteurs secondaires et tertiaires.}

Les errements traditionnels exigeaient et exigent encore dans l'esprit de nombreux utilisateurs ]e maintien de l'eau dans les fossés tertiaires à un niveau le plus élevé possible, presque égal à la cote des terrains. Les études pédo-agronomiques récentes ont montré au contraire que la revanche souhaitable était de $0,80 \mathrm{~m}$ à $1,00 \mathrm{~m}$, avec un écartement des fossés de l'ordre de $150 \mathrm{~m}$. La nature des terrains impose des talus de $3 / 2$ à $1 / 1$ et ces données conduisent à un fossé de $2,50 \mathrm{~m}$ à $3,00 \mathrm{~m}$ d'ouverture, pratiquement à fond plat, e'est-à-dire au fossé de 10 pieds traditionnel.

Les caractéristiques des canaux secondaires ont été déterminées en fonction des mêmes critères auxquels s'ajoute le souci de pallier par avance les conséquences d'un entretien médiocrement assuré.

\section{b) Canal principal.}

Le marais peut être considéré comme uniformément plat à une cote qui est inférieure de $0,50 \mathrm{~m}$ en moyenne à celles des fortes marées. Le franc d'eau souhaitable est de $0,80 \mathrm{~m}$ et, en se fixant une tolérance de $0,10 \mathrm{~m}$ autour de cette valeur, soit $+0,10 \mathrm{~m}$ à l'amont du réseau et $-0,10 \mathrm{~m}$, à l'aval, on se donne une pente, d'ailleurs faible, qui permet de calculer ce que devrait être la section du canal dans l'hypothèse du régime uniforme, pour le débit considéré à évacuer.

En fait, le régime n'est pas uniforme, en raison du jeu des marées qui elles-mêmes ont des amplitudes très variables. Il faut donc déterminer tout d'abord une marée moyenne type d'après les relevés marégraphiques existants.

L'analyse théorique du fonctionnement hydraulique peut se faire à partir de ces éléments, complétés par la courbe de remplissage du réseau dans l'hypothèse d'un écoulement nul à l'aval et en supposant constant l'apport d'amont. On peut par exemple utiliser la méthode décrite par Lencastre au congrès international des irrigations et du drainage à Madrid en 1960. Bien qu'elle suppose un certain nombre d'hypothèses simplificatrices, cette méthode qui fait intervenir les écoulements en résime varié peut encore être simplifiée tout en conservant une précision acceptable dans le cas particulier considéré.

Il n'est pas dans le propos de cet exposé d'entrer dans le détail de la méthode de calcul ainsi aménagée, mais seulement de faire ressortir les données qu'elle a permis de mettre en évidence et qui ont une influence sur le problème de l'entretien.

La première constatation a trait à l'importance des fossés tertiaires en ce qui concerne la capacité régulatrice du réseau. Dans le projet évoqué plus haut, ils représentent plus de $50 \%$ du volume tenu 
disponible pour stocker de l'eau en dehors des périodes d'écoulement, cependant que le total fossés tertiaires et canaux secondaires atteint $75 \%$, ce qui permet de limiter la remontée de l'eau à des valeurs très faibles compatibles avec un bon assainissement des terrains $(25$ à $35 \mathrm{~cm}$ selon les marées). Il est donc capital de maintenir ce réseau en bon état, c'est-à-dire pratiquement de conserver leur section aux fossés, bien qu'elle soit très surabondante au regard des strictes nécessités de l'écoulement.

En second lieu, les vitesses d'écoulement sont très faibles. Les terrains étant pratiquement plats, il faut donner de la pente aux canaux artificiellement el les possibilités sont limitées si l'on veut:

- conserver la revanche souhaitable sur le plan agronomique;

- limiter l'ouverture des émissaires dans la partie aval notamment.

Augmenter la pente en abaissant par exemple la cote du radier de l'ouvrage de sortie, donc celle de l'eau, aurait pour conséquence d'entraîner la fermeture plus rapide des écluses à marée montante, c'est-à-dire l'allongement de la période de fermeture. Au surplus, l'accélération de l'eau permet alors de réduire la section des ouvrages, mais indirectement aussi la capacité régulatrice du réseau, ce qui est incompatible a priori avec un long temps de fermeture.

Pratiquement, l'expérience montre, corroborée par le calcul, que les pentes ne peuvent ètre supérieures à 1 ou $2.10^{-4}$, ce qui représente des vitesses d'eau de l'ordre de $0,20 \mathrm{~m} / \mathrm{s}$. Dans cetle gamme de vitesses, le développement des herbes aquatiques au printemps est extrêmement abondant. En été, d'autre part, les réseaux fonctionnent rarement, le souci des riverains étant au contraire de conserver de l'eau. En définitive, le milieu se prête bien à une prolifération intense de plantes diverses, les unes que l'on trouve dans tous les sites aquatiques et les autres spécifiques des sites d'eau calme et dormante. Cette diversité des espèces végétales représentées complique singulièrement les problèmes de désherbage chimique qui seront évoqués plus loin.

En troisième lieu, les variations de niveau alternatives, surtout au voisinage des écluses de sortie, provoquent fréquemment des éboulements des talus, dont l'effet de comblement se joint à celui dû aux atterrissements, particulièrement intenses :

$1^{\circ}$ dans les fossés et canaux en eau douce, en raison des faibles vitesses d'écoulement;

$2^{\circ}$ dans les chenaux soumis au marnage, à l'aval des écluses. La mer el les eaux des estuaires du rivage Charentais sont en général très chargés en vase qui se dépose en quantités importantes au contact des eaux douces venues de l'amont.

Enfin, depuis quelques années, les exigences des cultures pratiquées dans certaines zones ne s'accomodent plus des aléas de l'assainissement gravitaire; il s'est avéré nécessaire de compléter leur équipement par des stations de relevage. Le réseau d'assainissement qui alimente la station doit évidemment être maintenu en parfait état, de manière à conserver ses caractéristiques hydrauliques d'ori- gine d'une façon plus impérative encore que dans le cas de l'assainissement gravilaire, pour des raisons évidentes. Mais il est nécessaire en outre que les fossés et canaux soient débarrassés aussi radicalement que possible des herbes aquatiques, faute de quoi, à la mise en route de la station, généralement en octobre, des masses énormes de végétation atteignant des dizaines de tonnes viennent l'engorger et en compromettre le fonctionnement.

Lutte contre les herbes aquatiques d'une part, enlèvement des atterrissements et reprise des talus d'autre part, constituent done les deux problèmes à résoudre. Les moyens manuels traditionnels tels que l'utilisation du faucard, de la chaine à faucarder ou de la bèche à long fer sont en voie de disparition faute de main-d'œuvre. On utilise maintenant :

- des techniques de désherbage chimique;

- des techniques de curage mécanique.

\section{Les techniques de désherbage chimique}

La mise en œuvre des techniques de désherbage chimique impose de bien connaître la flore à détruire, les produits phytocides existants et leur spectre actif. On déterminera ainsi le traitement à appliquer.

\subsection{Les plantes aquatiques.}

Bien que cette classification n'ait pas de valeur botanique, il est commode de distinguer :

2.1.1. Les plantes ImMERgéEs qui s'enracinent dans le fond du canal ou sur les berges et dont la tige est aérienne.

Les espèces dominantes appartiennent au groupe des monocotylédones : typha, rubaniers, phragmites ou roseaux et jones, qui se présentent en populations homogènes sur des étendues parfois très vastes. Dans les zones limitrophes des terres hautes, la flore est en général plus variée et les dicotylédones y occupent une place qui n'est plus négligeable.

2.1.2. Les plantes immergéEs dont l'appareil végétatif reste intégralement dans l'eau ou à la surface de l'eau. Les unes sont enracinées au fond et leurs feuilles s'étalent en surface tels les nénuphars, potamots, certaines renoncules. D'autres ont un appareil végétatif entièrement flottant, tels les lemna, hydrocaris. Enfin, une dernière catégorie comprend les plantes submergées qui utilisent pour leur nutrition et leur respiration les gaz dissous dans l'eau : cératophylle, myriophylle, élodéa canadensis, etc. Les algues ne sont que très rarement présentes.

Emergées ou immergées, les plantes aquatiques arrivent à former des masses considérables et à obturer complètement certains fossés ou canaux. 


\section{P. COULBOIS}

\subsection{Les produits désherbants.}

Les composés minéraux tels que le chlorate de soude ont été remplacés complètement par des dérivés organiques de synthèse que l'on peut classer en quelques grandes familles:

- Les acides organiques chlorés, tels que le dalapon et le basinex dont l'emploi est particulièrement indiqué sur les plantes émergées : typha, rubaniers, phragmites.

- Les auxines de synthèse, telles que l'acide 2-4 dichlorophénoxyacétique (2-4-D) et l'acide 2-4-5 trichlorophénoxyacétique $(2-4-5-T)$ ou l'acide méthychlorophénoxyacétique (M.C.P.A.) dont l'action est surtout notable sur les plantes dicotylédones à larges feuilles ainsi que sur les joncs et les plantes ligneuses. Les roseaux et graminées sont par contre assez résistants.

- L'aminotriazole, commercialisé sous diverses dénominations, particulièrement actif sur les plantes émergées, typha, rubaniers, phragmites, mais peu actif sur les joncs.

- Les urées substituées, commercialisées sous le nom de monuron et diuron, classées dans la rubrique des «désherbants totaux » et dont une application intéressante est l'épandage en qualité de stérilisant (cf. infra). Le diuron est également utilisé pour le traitement de la végétation immergée.

- Les ammoniums quaternaires, commercialisés sous le nom de diquat et de paraquat, qui sont des produits défoliants à action extrêmement rapide mais a remanence très faible; ils sont pratiquement les seuls à avoir une action notable sur la végétation immergée.

Ces divers produits peuvent ètre utilisés seuls, ou en association, soit pour détruire en un seul passage deux classes de plantes sensibles à deux produits distincts, soit pour exploiter l'effet de synergie que peut produire le mélange. Dans le premier cas, on peut citer en exemple le mélange dalapon $+2-4-\mathrm{D}$; dans le second, l'association aminotriazole + thyocyanate d'ammonium. Enfin, on ajoute parfois à la solution de produit phytocide un produit tensio-actif qui facilite la pénétration dans le végétal : la plupart des produits, quel que soit leur mode d'action ultérieur (désorganisation des assises génératrices, destruction de la chlorophylle ou autre), doivent en effet, pour être efficaces, pénétrer dans l'organisme végétal, lequel est fréquemment protégé des agressions extérieures par une cuticule cireuse.

\subsection{Les traitements.}

Compte tenu de la flore présente dans les fossés et canaux et des possibilités qu'offrent les divers produits commerciaux, les traitements types mis en œuvre dans le marais Charentais sont les suivants :

\subsubsection{VÉgÉtATION ÉMERGÉE.}

Dans le marais, où les espèces les plus fréquentes sont les phragmites, rubaniers et typhas, le produit systématiquement utilisé est l'aminotriazole activé à la dose de 1 à $2 \mathrm{~g} / \mathrm{m}^{2}$, épandu en solution dans l'eau. Il a été préféré aux dalapon et basinex, l'expérience ayant montré que son effet est plus durable. On peut admettre en effet que l'effet d'un traitement à $2 \mathrm{~g} / \mathrm{m}^{2}$ se fait sentir pendant les deux années suivantes et qu'un traitement d'entretien à demi-dose est suffisant la quatrième année.

Dans les zones bordant les terres hautes où la population est plus variée, l'utilisation d'un produit spécifique détruit l'équilibre qui s'est établi entre mono et dicotylédones. On a donc recours à un traitement complémentaire du premier, soit la même année, soit l'année suivante. On utilise également parfois un mélange de divers produits : aminotriazole, auxines de synthèse, atrazine, etc., de manière à réaliser une destruction aussi complète que possible en une seule application.

\subsubsection{VÉgétation IMMERGÉE.}

La destruction de la végétation émergée ne soulève plus de grosses difficultés dans l'état actuel des choses. Il n'en est pas de même avec la végétation immergée dont la destruction est difficile et l'éradication complète pratiquement impossible avec les moyens dont nous disposons. Cette situation est d'autant plus gènante que la disparition des plantes émergées entraîne en général le développement d'une flore immergée abondante dont la croissance n"est plus entravée par la couverture aérienne des plantes émergées.

Les seuls produits couramment utilisés sont le paraquat ou le diquat à la dose de 5 ppm de produit commercial, ce dernier étant le plus courant dans notre région.

Ce traitement appliqué à une population qui comporte par exemple une proportion de $50 \%$ de cératophylles pour $20 \%$ d'élodéa, $20 \%$ de potamots et $10 \%$ de divers entraine la disparition de la quasi-totalité de la végétation, mais le traitement doit être repris chaque année. Le paraquat serait utilisé au même titre que le diquat n'était son prix de revient plus élevé.

\subsubsection{LES TRAITEMENTS STERMLISANTS.}

Un essai de stérilisation effectué en 1960 et comportant l'application de $2 \mathrm{~g} / \mathrm{m}^{2}$ d'un mélange monuron + diuron sur le fond et les herges d'un canal neuf, suivi en 1961 d'un traitement identique à la dose de $1 \mathrm{~g} / \mathrm{m}^{2}$, avait permis de constater que les repousses de plantes émergées étaient négligeables pendant au moins quatre années. Cette technique prometteuse n'a malheureusement pas pu recevoir la diffusion qu'elle mérite; l'épandage du produit phytocide doit être fait sur un sol absolument sec, ce qui est la plupart du temps incompatible avec les nécessités du chantier, du moins en zone marécageuse.

Pour compléter ces éléments sur le désherbage chimique, il convient de donner quelques indications sur les conditions dans lesquelles doivent être faits les traitements et sur les limites de la méthode.

Le choix de l'époque du traitement est très important. Cette épociue est d'ailleurs variable d'une année à l'autre, car c'est le stade de maturité de la plante à détruire qui doit servir de repère. Pratiquement, les traitements ont lieu dans nos régions du 15 juin au 15 octobre, c'est-à-dire l'époque de la floraison-fructification. 
Selon la densité de la végétation, les doses de produit appliqué sur plantes émergées varient de 1,5 à $2,5 \mathrm{~g}$ par mètre carré traité et les quantités répandues de 1000 à 2500 litres par hectare. Le prix de revient de l'opération est de l'ordre de $0,10 \mathrm{~F} / \mathrm{m}^{2}$ avec un traitement à $2 \mathrm{~g} / \mathrm{m}^{2}$ d'aminotriazole (épandage compris). Sur plantes immergées, le diquat est mélangé à l'eau du canal dans la proportion de $5 \mathrm{ppm}$ (du produit commercial). Le prix de revient de l'opération est de $0,40 \mathrm{~F} / \mathrm{ml}$ pour un canal contenant environ $5 \mathrm{~m}^{3}$ d'eau au metre linéaire.

Les véhicules portant le matériel d'épandage doivent être du type tous terrains, car les conditions d'accès sont souvent difficiles. C'est ainsi que l'on dispose en Charente-Maritime d'un camion G.M.C. portant deux cuves de 8001 et équipé d'une lance alimentée par un groupe moto-pompe pouvant donner une pression de $30 \mathrm{~kg}$. On obtient de la sorte une bonne pulvérisation et un jet suffisamment puissant pour pénétrer les masses de végétation parfois considérables à traiter. Le même matériel est utilisé pour le traitement des plantes immergées, le jet de pulvérisation assurant un bon brassage du diquat dans l'eau du canal.

Les auteurs anglo-saxons, qui utilisent le terme de « contrôle de la végétation aquatique » pour désigner ces techniques chimiques, mettent ainsi bien en valeur les limites de la méthode. La destruction de l'équilibre préexistant au traitement entraine des modifications, notamment des inversions de flore souvent imprévisibles qui nécessitent un ajustement permanent des traitements à mettre en œuvre. Enfin, bien que la toxicité des produits utilisés sur les animaux inférieurs (poissons et animaux dont ils se nourrissent) et supérieurs (bovins et chevaux) soit très faible aux doses couramment utilisées, il n'en demeure pas moins les risques, d'ailleurs inappréciables actuellement, que fait peser sur le milieu la modification d'un équilibre biologique.

\section{Les techniques} de curage mécanique

Bien que très concurrencé par les traitements chimiques, il existe un procédé de destruction mécanique de la végétation aquatique qui se maintient encore dans le marais Charentais : le bateau faucardeur.

Le batean faucardeur, dont il existe de nombreux modèles, est un engin à fond plat propulsé par roue ou hélice et muni d'une ou plusieurs lames de faucheuses classiques qui peuvent en général prendre des inclinaisons variables de manière à couper les herbes poussant dans le fond et sur les berges. Après passage du bateau, il faut assurer l'évacuation des herbes, ce qui est facile dans les rivières à fort courant et qui est réalisé par des chasses à marée basse dans les marais côtiers.

Mais lorsqu'on évoque le curase par des moyens mécaniques, on pense surtout aux machines per- mettant le nettoyage complet des fossés et canaux, l'enlèvement simultané de la végétation et des atterrissements. La pelle mécanique est alors le premier engin dont l'utilisation vient à l'esprit et il est certain que moyennant quelques aménagements, notamment l'équipement avec des godets spéciaux très larges, elle peut convenir parfaitement. Le principal obstacle à son utilisation pour les fossés de 10 pieds, où le cube de déblais à extraire est faible, est le prix de revient élevé de l'opération. Il s'y ajoute que les déblais extraits sont déposés en cordons le long du canal, qu'ils me peuvent ètre régalés qu'un ou deux ans plus tard après dessication et que, pendant ce temps, ils gènent l'exploitation de la parcelle riveraine ef son assainissement.

Si donc l'utilisation de la pelle mécanique modifiée demeure la règle pour les canaux d'ouverture supérieure à $4 \mathrm{~m}$, il faudrait pouvoir disposer pour l'entretien des fossés tertiaires de 10 pieds d'un engin répondant aux critères suivants :

- être léger et maniable de manière à pouvoir accéder aux endroits difficiles et à pouvoir circuler sur routes;

- travailler « en continu», soit dans le fond du fossé, soit sur le bord;

- exécuter en un seul passage l'extraction et l'épandage des déblais extraits et bien entendı réaliser ces opérations dans des conditions économiques acceptables.

Enfin, l'entretien des chenaux soumis au marnage pose un problème encore différent en raison des difficultés d'accès et de la faible portance des terrains riverains d'une part, de la nature des vases extraites d'autre part, problème qui ne saurait être résolu techniquement et économiquement par l'utilisation de la pelle mécanique.

Compte tenu de l'ensemble de ces considérations, les moyens mécaniques mis en œure actuellement dans le marais Charentais sont les suivants :

\subsection{La suceuse.}

Les engins utilisés dérivent directement du type travaux publics, mais sont en général de plus faible taille pour pouvoir accéder à des chenaux ou canaux de faible largeur. Rappelons que la suceuse se compose d'un ponton flottant portant à l'extrémité d'un bras mobile un système dilacérateur et désagrégateur. Le mélange eau-déblais est ensuite aspiré par une pompe et rejeté sur un terrain d'épandage voisin qui doit être endigué soigneusement pour éviter le retour des déblais au canal.

L'opération nécessite un volume d'eau important, environ sept fois le volume de déblais à extraire, ce qui représente une lourde sujétion s'ajoutant à celle de l'épandage. En définitive, la suceuse n'est utilisée que lorsque l'accès par voie de terre est impossible et que les arbres plantés sur le bord des canaux empêchent le passage et le travail en continu d'un engin terrestre. C'est le cas :

-- du marais poitevin et des marais de fonds de vallée en général;

- des chenaux soumis au marnage. 


\subsection{Le bac à rateau.}

C'est un bateaut à fond plat muni à l'avant d'une forme repliable, en bois ou en tôle, qui épouse grossièrement la forme trapézoïdale du canal. La partie inférieure de la forme peut être munie de dents.

Une fois dépliée, la forme obture en partie le canal, créé donc une perte de charge localisée, donc une dénivelée entre amont et aval du bateau. Lorsque la charge est sufisamment importante pour vaincre le frottement, le bateau est poussé vers laval el la forme racle le fond, mettant en suspension les atterissements et arrachant les herbes qui sont entrainées par le courant.

Le bac à rateau est utilisé systématiquement dans les chenaux sommis au marnage el dans les biefs avals des grands canaux ou l'influence de la marée est appréciable. Tout simple qu'il soit, cet appareil rend les plus grands services.

\subsection{Les cureuses rotatives.}

Il existe en France d'innombrables modèles de cureuses rotatives qui diffèrent par la nature de leurs pièces travaillantes : vis hélicö̈dales, cylindriques ou coniques, disques ou chaines à palettes, etc., et par leur position de travail : latéralement, à cheval ou dans le fond du fossé. Elles répondent dans leur principe aux exigences formulées plus haut c'est-à-dire qu'elles permettent l'extraction des déblais et de la végétation et leur ejection latérale sur une largeur suffisante pour éviter la formation d'un bonrelet. Les deux opérations peuvent être réalisées par le même organe ou par deux organes différents suivant les cas. Des dispositifs fixes, genre socs ou coutres, permettent en outre de retaluter le fossé à son gabarit. Ces machines ont généralement été concues pour I'entretien de petits fossés type fossés routiers ou rigoles d'assainissement et aucune d'elles ne peut s'adapter au marais Charentais pour résoudre son problème essentiel: l'entretien des fossés de 10 pieds.

Les constructeurs allemands, par contre, ont mis au point depuis plusieurs années une série de cureuses rolatives destinées à l'entretien des fossés de marais bordant la mer du Nord el la Ballique.

Malheureusement encore, ces machines sont conçues pour travailler dans des fossés de gabarit légèrement plus faible, dans des terrains généralement plus légers et l'expérience a montré qu'elles ne pouvaient être utilisées telles quelles.

Plusieurs d'entre elles ont paru cependant dans leur principe répondre aux exigences propres du marais Charentais, ee qui a conduit a faire construire localement des engins qui en dérivaient par extrapolation.

La première cureuse importée vers 1956 provenait des établissements Ritscher de Hambourg. L'organe travaillant était une vis hélicoüdale sous carter; les déblais el les vases raclés par une lame passant dans le fond du fossé élaient repris par la vis, remontés jusqu’à un éjecteur à palette et projetés à $20 \mathrm{~m}$ du bord du fossé. C'ensemble était porté par un tracteur semi-chenillé de $40 \mathrm{ch}$.

Son extrapolation, un monstre de quinze tonnes porté sur chassis chenillé, dut être abandonné en raison de sa taille et de son poids.
En 1959, apparut la machine Wulff fabriquée par les établissements Johannes Flügger, petit constructeur de Delmenhorst près de Brème. Elle comporte:

- une vis hélicoïdale à bord tranchant de $0,50 \mathrm{~m}$ de longueur disposée horizontalement dans le fond du fossé;

- un coutre circulaire monté fou sur un arbre;

- un éjecteur circulaire à 4 pales de $0,80 \mathrm{~m}$ de diamètre.

L'ensemble ainsi constitué est porté à l'extrémité d'un bras en trois éléments qui prend appui sur le boitier de prise de force du tracteur support (en l'occurrence un tracteur ma.N $50 \mathrm{ch}$ ) et autour duquel il est relevable. Ce bras est constitué d'un tube à l'intérieur duquel passe l'arbre de transmission communiquant le mouvement de la prise de force à l'éjecteur et à la vis.

En position de travail, la tête est descendue dans le fossé et la vis s'y trouve disposée horizontalement dans le fond, l'éjecteur et le coutre étant parallèles au talus (l'inclinaison est réglable). La vis ramène vers le bord la vase et les herbes dilacérées ainsi que les produits de retalutage découpés par le coutre et l'éjecteur projette le tout latéralement sur le bord.

Cette machine est très intéressante par sa légèreté et sa simplicité mécanique; elle atteint des rendements de 2 à $3 \mathrm{~km}$ par jour en Allemagne $\grave{a}$ des prix de revient très acceptables et c'est ce qui a déterminé à réaliser un modèle plus «étoffé » s'en inspirant largement.

La dernière cureuse créée en 1964 est portée par un tracteur type forestier à quatre roues motrices et directrices, de $120 \mathrm{ch}$, équipé d'une boite de vitesses auxiliaire permettant un avancement en première réduite de $400 \mathrm{~m} /$ heure. Les pièces travaillantes sont montées à l'extrémité d'un bras relevable prenant appui sur l'arrière du tracteur et comportant :

$1^{\circ}$ un disque de fond en forme de calotte sphérique crénelé, type Cover-crop, de diamètre $0,75 \mathrm{~m}$ destiné à découper la végétation en place et à repousser les vases de fond et cette végétation vers le bord du fossé. Il tourne à $250 \mathrm{tr} / \mathrm{mn}$ autour d'un axe qui est horizontal lorsque la machine travaille et qui fait un angle de $60^{\circ}$ avec le sens d'avancement. La concavité du disque est tournée vers l'avant;

$2^{\circ}$ un disque latéral destiné à retailler les irrégularités du talus à sa partie supérieure et qui est monté fou sur un arbre;

$3^{\circ}$ un éjecteur à palette de $1,20 \mathrm{~m}$ de diamétre, tournant à $250 \mathrm{tr} / \mathrm{mn}$, qui est, comme le coutre, parallèle au talus en position de travail et qui retalute la partie inférieure du fossé et expulse les déblais et débris végétanx extraits.

Toutes les transmissions sont hydrauliques: mouvements du bras par vérins, entrainement du disque crénelé et de l'éjecteur respectivement par deux moteurs de 20 et $60 \mathrm{ch}$. Une pompe générale fournit l'huile sous pression qui est stockée dans un réservoir de $600 \mathrm{l}$.

Les rendements que l'on peut espérer obtenir sont de l'ordre de $60 \mathrm{~m} /$ heure de fossé curé avec deux passages sur chaque bord. En fait, on pourrait 
limiter les passages à deux, un sur chaque bord, les deux derniers passages ne servant qu'à donner un fini qui n'ajoute rien à l'intérêt de l'opération. Le rendement pourrait dans ces conditions atteindre 100 à $120 \mathrm{~m}$ /heure. Il faut préciser cependant que la machine ne permet pas de curer les angles des fossés, qui doivent être terminés à la main. Il reste également à apprécier la tenue mécanique d'un ensemble relativement très complexe, qui s'avère a priori très délicate et nécessiterait sans doute la révision de certains montages.

\section{Les méthodes d'entretien}

Les données techniques de l'entretien des réseaux d'assainissement doivent être mises en balance avec les contraintes liées à leur fonctionnement hydraulique qui ont été exposées plus haut et également avec les impératifs d'ordre économique. Ces derniers, par exemple, font rejeter $a$ priori l'idée d'une méthode d'entretien qui conduirait chaque année à remettre dans l'état idéal les fossés et canaux. Le processus d'envasement est plus ou moins rapide selon les réseaux, mais jamais au point de nécessiter un curage annuel. Quant à la végétation, on parvient à la « contrôler $\gg$ en utilisant des produits rémanents, assortis de quelques traitements d'entretien plus espacés.

L’idée générale à retirer de ces considérations et de dix ans d'expérience est que la solution la moins mauvaise au problème de l'entretien réside actuellement dans l'utilisation conjointe des techniques mécaniques et de désherbage chimique.

L'entretien sera donc assuré par le passage à intervalles réguliers d'un engin de curage mécanique et, dans l'intervalle séparant deux curages, par le contrôle au plus bas niveau possible de la végétation aquatique. Accessoirement, cette der- nière précaution évitera les inconvénients déjà signalés dans le cas où le réseau alimente une sta. tion d'exhaure; elle facilite également le travail ds la cureuse en évitant le bourrage des pièces rota. tives par une végétation trop abondante.

Dans le cas des grands fossés el des canaux, la pelle mécanique est de règle et le cycle d'entretien est de dix ans environ.

Les chenaux soumis au marnage sont curés chaque année à l'aide du bac à rateau. La végétation ne constitue généralement pas un problème dans ce cas.

Dians les marais de fonds de vallée, la présence des arbres sur le bord des fossés et canaux implique une utilisation quasi obligatoire de la pelle mécanique.

Enfin, pour les petits fossés des marais côtiers, on avait le choix a priori entre un cycle relativement court, de l'ordre de six ans, avec passage de la cureuse, ou l'allongement du cycle à dix ans, l'augmentation du cube d'atterrissement obligeant à l'emploi de la pelle mécanique.

Pour des raisons techniques évidentes, la première solution parait la meilleure. Son prix de revient peut être évalué d'autre part de la manière suivante, en admettant que le cycle comportera également un traitement chimique de base et un traitement d'entretien :

- 1 désherbage complet ........ $0,30 \mathrm{~F} / \mathrm{ml}$

- 1 traitement d'entretien ....... 0,15

- 1 curage mécanique $\ldots \ldots \ldots \ldots, 0,77$

$1,22 \mathrm{~F} / \mathrm{ml}$

soit en moyenne $0,20 \mathrm{~F} / \mathrm{ml}$.an. Le curage décennal à la pelle revient à $2,75 \mathrm{~F} / \mathrm{ml}$, soit une incidence de $0,275 \mathrm{~F} / \mathrm{ml}$.an. L'avantage est encore sur ce plan à la méthode que l'on peut qualifier d'entretien continu. Elle devrait ètre systématiquement appliquée dans l'avenir si les espoirs mis dans la dernière cureuse construite ne sont pas déçus.
M. le Président remercie $M$. Coulbors de son exposé fort intèressant et très heureusement illustré.

M. Hauser aimerait avoir quelques renseignements sur les prix d'entretien des canaux. D'autre part, il demande à M. Covruors s'il peut formuler des conseils de nature à influer sur le choix des sections-types de canaux neufs en vue de faciliter leur entretien ultérieur.

En ce qui concerne les prix d'entretien. M. Courbors renvoie les personnes intéressées aux chiffres qui figurent dans sa communication, et qu'il résume comme suit :

- pour un cycle de cinq ans qui comprend un désherbage complet, un traitement d'entretien et un curage meeanique, on compte $1,22 \mathrm{~F}$ par mètre linéaire, soit sur six ans, environ $0,20 \mathrm{~F}$ par metre et par an;

- pour un curage à la pelle, tous les dix ans, on arriverait à une valeur theorique de 0,27 ou $0,30 \mathrm{~F}$ par mètre lineaire et par an.
Les prix varient notablement d'un cas à l'autre.

A propos des formes et des tracés a donmer aux canaux pour faciliter leur entretien, M. Covruors rappelle qu'il a traité de l'entretien de réseaux existant depuis deux ou trois cents ans. Il n'y a, actuellement, aucune solution universelle qui permette, au moment de l'etablissement d'un réseau neuf, d'optimiser les dépenses d'entretien : on en est réduit à utiliser les mêmes gabarits de fossés, les mêmes pentes, les mêmes systèmes d'assainissement qu'autrefois.

Il semble, a priori, que l'utilisation d'engins du type pelle mécanique revienne fort cher. Il faut noter, toutefois, qu'il existe sur le marché des appareils, utilisables pour les fossés routiers, qui permettent le curage de petites rigoles de 0,90 à $1,20 \mathrm{~m}$ à des profondeurs de 0,60 ou $0,70 \mathrm{~m}$; ils mériteraient d'être essayés dans le domaine qui nous occupe.

A la demande de M. le Président, M. Coulbous dit qu'il n'a pas connaissance de l'existence de matériels russe ou 


\section{P. COUlBoIs}

américain; le marché des cureuses et d'ailleurs assez limité.

M. Nicolo demande quels sont les problemes posés par la toxicité des produits utilisés pour le désherbage chimique et la pollution des eaux qui en résulte.

M. Counzors fait observer qu'il y a deux sortes de produits; les uns sont instantanement décomposés, il n'y a donc pas toxicite; les autres, comme les acides chlorćs, ont une plus grande rémanence; mais aux doses utilisées, les animaux n'en pâtissent pas. De toute façon, pour chaque produit, on procède à des expériences (sur une populaition de vairons, par exemple), dans des conditions artificielles qui permettent de déterminer la concentration correspondaint au senil de toxicite. Tontefois, les doses toxiques s'ajoutent chez les poissons, du fait de leur accumulation daus les tissus graisseux; cela semble comporter certains risques; aussi les Hollandais font-ils preuve d'une grande prudence dans l'emploi des désherbants.

M. Gannels fait part de quelques réflexions qui lui sont venues a l'esprit à la suite de l'exposé remarquable de M. Coulbois :

« $1^{\circ}$ Le système actuel de fossés relativement larges, a faible pente, a faible écoulement favorise la prolifération d'herbes aquatiques et rend l'entretien assez coûteux.

En fonction du problème réel à résoudre et des techniques nouvelles en matière d'aménagements hydro-agricoles, doit-on considérer le système ancien comme périmé et ponrait-on concevoir des systèmes phus modernes et moins oncirenx d'entretien?

$\ll 2^{\circ}$ Quelle est l'organisation des agriculteurs qui prend en charge les travaux d'entretien des réseaux de fossès?

« $3^{\circ}$ Ne pourrait-on pas, dans certains cas, recourir aux lance-1lammes, après assèchement des fossés en été?
« 4" Y-a-t-il en Charente une influence, sur l'entretien des canaux, des rejets d'installations industrielles?

İn réponse a ces questions, M. Couluors donne les précisions ci-après :

« 1" La conception du réseau d'assainissement est liee à l'utilisation du marais. Le systeme actuel est acceptable dans le cadre d'une utilisation extensive du marais en prairie naturelle. Les essais en cours de réalisation tendent à lui substituer un assainissement beaucoup plus profond, enterré a $0,90-1,00 \mathrm{~m}$, en vue notamment d'assurer un dessalage du sol. Dans ces conditions, la conception et le tracé des l'éseaux risquent d'évoluer profondément, sans qu'on puisse espérer de ces modifications un allègement des charges d'entretien, au contraire;

"2* Il existe des associations syndicales de propriétaires (environ 130) qui gèrent des périmètres variant de 25 ha à 3000 ha : elles sont fédérées en un syndicat mixte qui dispose d'une régie de travaux, équipée avec des pelles et des engins d'entretien (cureuses et pulvérisateurs). C'est l'UNIMA qui a fait les frais des désherbants chimiques d'une part, et par curage mécanique d'autre part. L'Unima a dépensé des sommes considérables pour l'achat de matériels étrangers, puis pour la mise au point de prototypes extrapolés de ces matériels et enfin pour leur essai;

«3 $3^{\circ}$ L'assèchement des fossès en été est impossible en laison des exigences formulées par les utilisateurs. Quant a l'utilisation du lance-flammes, l'expérience a été faite, mais on a dû renoncer définitivement à ce système, car il nécessite des quantités de carburants beaucoup trop importantes, est dangereux et d'utilisation difficile;

« $4^{\circ}$ Les pollutions d'origine urbaine et industrielle n'ont en général, pas d'influence sur les canaux qui nous occupent, car ceux-ci se trouvent en zone essenticllement agricole.»

\section{Abstract \\ Drainage ditch operation and maintenance in the Charente-Maritime marshes by P. Coulbois *}

The formation and structure of the Charente-Maritime marshes are described and the leading features of their drainage networks stated.

This is followed by a brief analysis of the governing ditch design principles and network operating characteristics, especially those having a direct bearing on maintenance requirements, i.e. the following:

(i) The importance of the tertiary ditch network as regards the regulating capacity of the network, in which the flow is tidal;

(ii) The low flow velocities, which are conductive to weed growth;

(iii) Water level variations due to the intermittent flow.

The main ditch maintenance methods are then described, i.e. the following:

\section{Chemical weed destruction.}

The most important weeds to destroy are listed, also products in current use and the most frequent forms of treatment, i.e. treatment of the plants in situ above or under water, and sterilization.

\section{Mechanical weeding.}

The main types of mechanical ditch-clearing equipment are brieffy reviewed, including mechanical shovels, suction dredgers, helical clearers, etc. The author dwells at length on special equipment of the helical type made in Germany and prototypes of similar equipment produced in the Charente-Maritime region.

The report closes with a few words on maintenance methods for wide ditches, tidal ditches and small ditches, the common feature of which is that they all involve the use of chemical weed-killing techniques and mechanical maintenance equipment.

* Ingénieur du Génie Rural, des Eaux et des Forêts, à la Direction départementale de l'Agriculture de la Charente-Maritime. 